\title{
Electronic medical record data to identify variables associated with a fibromyalgia diagnosis: importance of health care resource utilization
}

This article was published in the following Dove Press journal:

Journal of Pain Research

5 March 2015

Number of times this article has been viewed

\author{
Elizabeth T Masters' \\ Jack Mardekian' \\ Birol Emir ${ }^{\prime}$ \\ Andrew Clair \\ Max Kuhn ${ }^{2}$ \\ Stuart L Silverman ${ }^{3}$ \\ 'Pfizer, Inc., New York, NY, 2Pfizer, Inc., \\ Groton, CT, ${ }^{3}$ Cedars-Sinai Medical \\ Center, Los Angeles, CA, USA
}

Background: Diagnosis of fibromyalgia (FM) is often challenging. Identifying factors associated with an FM diagnosis may guide health care providers in implementing appropriate diagnostic and management strategies.

Methods: This retrospective study used the de-identified Humedica electronic medical record (EMR) database to identify variables associated with an FM diagnosis. Cases $(n=4,296)$ were subjects $\geq 18$ years old with $\geq 2$ International Classification of Diseases, Ninth Revision (ICD-9) codes for FM (729.1) $\geq 30$ days apart during 2012, associated with an integrated delivery network, with $\geq 1$ encounter with a health care provider in 2011 and 2012. Controls without FM (no-FM; n=583,665) did not have the ICD-9 codes for FM. Demographic, clinical, and health care resource utilization variables were extracted from structured EMR data. Univariate analysis identified variables showing significant differences between the cohorts based on odds ratios (ORs).

Results: Consistent with FM epidemiology, FM subjects were predominantly female (78.7\% vs $64.5 \% ; P<0.0001$ ) and slightly older (mean age 53.3 vs 52.7 years; $P=0.0318$ ). Relative to the no-FM cohort, the FM cohort was characterized by a higher prevalence of nearly all evaluated comorbidities; the ORs suggested a higher likelihood of an FM diagnosis $(P<0.0001)$, especially for musculoskeletal and neuropathic pain conditions (OR 3.1 for each condition) Variables potentially associated with an FM diagnosis included higher levels of use of specific health care resources including emergency-room visits, outpatient visits, hospitalizations, and medications. Units used per subject for emergency-room visits, outpatient visits, hospitalizations, and medications were also significantly higher in the FM cohort $(P<0.0001)$, confirming resource utilization as an important variable associated with an FM diagnosis.

Conclusion: Significant differences between the FM and no-FM cohorts were observed for nearly all the demographic, clinical, and health care resource variables, suggesting an association with FM diagnosis. These results also support use of EMR data for identifying variables associated with FM, which may help in the diagnosis and management of this condition.

Keywords: retrospective database analysis, predictors, musculoskeletal pain, observational study, real world data

\section{Background}

Widespread pain is the hallmark symptom of fibromyalgia (FM), a chronic, complex musculoskeletal condition that remains undiagnosed in a substantial proportion of patients with this disease. ${ }^{1}$ While the pain is generally defined as bilateral both above and below the waist and includes axial skeletal pain, ${ }^{2} \mathrm{FM}$ is also characterized by a variety of other symptoms and comorbidities, including fatigue, sleep and mood disturbances, cognitive dysfunction, and irritable bowel syndrome. ${ }^{3}$ The substantial socioeconomic
Correspondence: Elizabeth T Masters Pfizer, Inc., 235 East 42nd St,

New York, NY I0017, USA

Tel +l 2127332682

Fax +l 2129737343

Email elizabeth.masters@pfizer.com 
burden of FM is well recognized, resulting from reductions in patient function, productivity, and quality of life, as well as high health care resource utilization. ${ }^{4-8}$

The 1990 American College of Rheumatology (ACR) classification criteria have long provided guidance for the diagnosis of FM based on the primary criteria of widespread pain and a manual tender-point exam for demonstrating the presence of tenderness at a minimum of 11 of 18 specified points. These criteria were updated in 2010 by adding a symptom-severity assessment and eliminating the tenderpoint exam. ${ }^{4}$ Despite the availability of these diagnostic criteria and various screening tools, FM patients still cycle through the health care system for several years before receiving a diagnosis; patients have reported delays of up to 5 years from when they first presented to a physician about their FM symptoms to receiving an actual diagnosis. ${ }^{1,3}$

Identifying variables associated with, or predictive of, developing FM can enhance understanding of the disease and provide a focus for development of appropriate management strategies. Previously, patient-reported registry data demonstrated that the widespread pain that is characteristic of FM was predicted by somatic symptoms, psychosocial and socioeconomic factors, fatigue, sleep problems, and workplace stress. ${ }^{5}$ A recent narrative review of biologic markers further highlighted the need for such identification, since while several markers can be considered suggestive of FM, none has unequivocally been shown to be a clinically useful predictor of developing this condition. ${ }^{6}$

Another relevant approach is to identify variables associated with an FM diagnosis, which can be used to inform health care providers of patients in the clinical setting who have a high likelihood of FM, thus reducing the diagnostic delay and facilitating earlier treatment initiation. To identify such variables, it may be necessary to go beyond the demographic and clinical characteristics that have been evaluated based on their ready availability in databases that are frequently used for these types of analyses.

The emergence of electronic medical records (EMR) may provide an opportunity to evaluate a wider range of variables associated with an FM diagnosis in the clinical setting. EMR capture real-world, patient-level data that represent integral components of provider care. ${ }^{7}$ These records allow a more complete longitudinal evaluation, including variables that may not necessarily be available from other sources such as claims databases or patient registries. Consequently, results generated using EMR may have greater applicability to clinical practice, and retrospectively evaluating "EMR markers" to characterize the variables associated with an eventual FM diagnosis may provide an opportunity for the earlier identification of FM patients. Therefore, the purpose of the study reported here was to evaluate EMR data with regard to differences in demographic, clinical, and health care resource utilization variables between subjects with a diagnosis of FM and those without FM. Such characterization may provide a first step toward identification of variables predictive of an FM diagnosis to help support the clinical management of FM.

\section{Methods \\ Data source}

This retrospective study was designed to use structured EMR data from the Humedica database to evaluate the characteristics associated with FM diagnosis. The database has broad geographic representation across the USA and captures a range of information longitudinally including demographic, clinical, claims, and medical administrative information. ${ }^{8}$ Humedica aggregates de-identified EMR data from providers across the continuum of care. Structured data include demographic information, clinical characteristics, and health care resource utilization. All records are linked through a unique patient identifier and are fully Health Insurance Portability and Accountability Act compliant with regard to patient and provider identification as well as protected health information.

\section{Subjects}

All subjects who met inclusion and exclusion criteria were included in the study. Subjects were required to be $\geq 18$ years of age in 2011, and enrolled in an integrated delivery network with at least one encounter with a health care provider recorded in the Humedica database in both 2011 and 2012. Subjects were excluded if they had at least one medical claim anytime during 2011-2012 with an International Classification of Diseases, Ninth Revision, Clinical Modification (ICD-9-CM) diagnosis code for malignant cancer (except for basal-cell and squamous-cell skin cancers and benign neoplasms) or an International Classification of Diseases, Ninth Revision (ICD-9) code for diagnosis or procedure for transplantation. Subjects who were resident in a nursing inpatient facility anytime during 2011-2012 were also excluded, as were subjects with evidence of an FM diagnosis (ICD-9 code 729.1) prior to 2012.

An FM cohort was identified from among subjects who met all inclusion and exclusion criteria, and was defined based on the presence of at least two ICD-9 codes for FM (729.1; myalgia and myositis, unspecified) $\geq 30$ days apart during 2012; this 
code is used in database analyses in the absence of a specific ICD-9 code for FM. The no-FM cohort consisted of similar subjects but without the FM ICD-9 codes.

\section{Analyses}

The EMR database captures information documented by health care providers at the point of care for patients actively seeking and receiving care. Variables included demographic and clinical characteristics reported by patients and clinicians, general comorbidities defined based on ICD-9 codes documented in the EMR, the Deyo-Charlson Comorbidity Index, ${ }^{9}$ and health care resource utilization for the year 2011 (ie, prior to the FM diagnosis) across a variety of categories including inpatient and outpatient encounters, visits in which diagnostic/laboratory testing or imaging was ordered, and medications. Humedica aggregates resource utilization information from health care delivery encounters, and this information was used to estimate resource use by encounter type. These characteristics for the FM and no-FM cohorts were summarized descriptively, with comparisons performed using likelihood ratio chi-square tests for categorical variables and two-sample $t$-tests for continuous variables. Univariate logistic regression analysis was performed to identify variables showing significant differences between the populations with and without an FM diagnosis based on estimated odds ratios (ORs) and their associated $95 \%$ confidence intervals ( $95 \%$ CIs). All analyses were conducted using SAS software (v 9.2; SAS Institute Inc., Cary, NC, USA).

\section{Results}

\section{Patient characteristics}

From among the individuals in the Humedica de-identified database during 2011 and $2012(\mathrm{~N}=9,318,581)$, a population was identified of 587,961 subjects meeting all inclusion/ exclusion criteria and having all required demographic and clinical information (details of population attrition are presented in Table 1). From this population, 4,296 subjects $(0.7 \%)$ were identified as having FM based on the predefined ICD-9 code criteria (ie, $\geq 2$ FM codes), with 583,665 subjects remaining in the no-FM cohort. During 2012, there were 7,157 subjects $(1.2 \%)$ who met inclusion and exclusion criteria but had only one FM ICD-9 code, and these subjects were included in the no-FM cohort.

As shown in Table 2, there were significant differences between the cohorts with regard to demographic characteristics, including a higher predominance of females in the FM
Table I Sample attrition table

\begin{tabular}{ll}
\hline Attrition criterion & $\mathbf{N}$ \\
\hline $\begin{array}{l}\text { Total number of de-identified patients in the Humedica } \\
\text { database available for this research from January I, }\end{array}$ & $9,3 \mid$ I8,58I \\
20I I-December 3I, 20I2 & \\
Patients aged $\geq \mid$ I years in 20II & $7,696,733$ \\
$\quad$ And enrolled in integrated delivery network & $4,192,869$ \\
$\quad$ And with $\geq$ I encounter with a health care provider & 720,912 \\
in 20II and 20I2 & \\
$\quad$ Patients with cancer diagnosis (exclusion) & 109,094 \\
$\quad$ Patients with transplantation (exclusion) & 5,163 \\
$\quad$ Patients with nursing home (exclusion) & 5,099 \\
$\quad$ Patients with FM diagnosis prior to 20I2 (exclusion) & 20,026 \\
Excluding patients with cancer diagnosis, transplantation, & 132,574 \\
in nursing home, or FM diagnosis prior to 20I2 & \\
Patients meeting all inclusion and exclusion criteria & 588,338 \\
$\quad$ Missing sex value & 377 \\
Total patients in analysis population & 587,96 I \\
$\geq 2$ ICD-9 codes for FM (729.I) at least 30 days apart & 4,296 \\
during 20I2 & \\
Number of patients in no-FM cohort & 583,665 \\
\hline
\end{tabular}

Abbreviations: FM, fibromyalgia; ICD-9, International Classification of Diseases, Ninth Revision.

Table 2 Demographic characteristics of the evaluated cohorts

\begin{tabular}{|c|c|c|c|}
\hline \multirow[t]{2}{*}{ Variable } & \multicolumn{2}{|l|}{ Value } & \multirow[t]{2}{*}{$P$} \\
\hline & $\begin{array}{l}F M \\
(n=4,296)\end{array}$ & $\begin{array}{l}\text { No-FM } \\
(n=583,665)\end{array}$ & \\
\hline Female, n (\%) & $3,379(78.7)$ & $376,653(64.5)$ & $<0.000$ I \\
\hline Age, years, mean (SD) & $53.3(14.6)$ & $52.7(16.3)$ & 0.0318 \\
\hline Age distribution, n (\%) & & & $<0.000$ I \\
\hline $18-49$ years & I,65I (38.4) & $229,910(39.4)$ & \\
\hline $50-64$ years & $\mathrm{I}, 482(34.5)$ & $|83,4| 4(3 \mid .4)$ & \\
\hline$\geq 65$ years & I, $163(27.1)$ & $|70,34|(29.2)$ & \\
\hline Race, n (\%) & & & $<0.0001$ \\
\hline African American & $296(6.9)$ & 83,727 (14.3) & \\
\hline Asian & $32(0.7)$ & II,294 (I.9) & \\
\hline Caucasian & $3,778(87.9)$ & 429,955 (73.7) & \\
\hline Other/unknown & $190(4.4)$ & $58,689(10.1)$ & \\
\hline \multicolumn{4}{|l|}{ Region, n (\%) } \\
\hline Midwest & $2,540(59.1)$ & $375,872(64.4)$ & $<0.0001$ \\
\hline Northeast & $373(8.7)$ & I I8, I 46 (20.2) & \\
\hline South & $\mathrm{I}, \mathrm{I} 25(26.2)$ & $75,4 \mid 4$ (I2.9) & \\
\hline West & $5(0.1)$ & $458(0.1)$ & \\
\hline Other/unknown & $253(5.9)$ & I3,775 (2.4) & \\
\hline Insurance type, n (\%) & & & $<0.000$ I \\
\hline Commercial & |8| (4.2) & I45,425 (24.9) & \\
\hline Medicaid & $7(0.2)$ & $3,740(0.6)$ & \\
\hline Medicare & $88(2.0)$ & $61,151(10.5)$ & \\
\hline Missing/unknown & $4,017(93.5)$ & $370,332(63.4)$ & \\
\hline Other payer type & $0(0.0)$ & $297(0.1)$ & \\
\hline Uninsured & $3(0.1)$ & $2,720(0.5)$ & \\
\hline $\begin{array}{l}\text { Charlson Comorbidity Index, } \\
\text { mean (SD) }\end{array}$ & $0.8(1.3)$ & $0.5(I .1)$ & $<0.0001$ \\
\hline
\end{tabular}

Abbreviations: FM, fibromyalgia; SD, standard deviation. 
cohort $(78.7 \%$ vs $64.5 \% ; P<0.0001)$ and differences in age and race as well as geographic distribution and insurance plans. Subjects in the FM cohort also had a significantly higher score on the Charlson Comorbidity Index ( 0.8 vs 0.5 ; $P<0.0001$ ) (Table 2).
In the year prior to the FM diagnosis, nearly all evaluated general comorbid conditions had a significantly higher prevalence in the FM cohort relative to the no-FM cohort (Table 3).

Univariate analysis showed that nearly all comorbidities were associated with a higher likelihood of an FM

Table 3 General comorbidity prevalence of the evaluated cohorts during $201 \mathrm{I}$ and univariate odds ratios (ORs) for a fibromyalgia (FM) diagnosis

\begin{tabular}{|c|c|c|c|c|}
\hline \multirow[t]{2}{*}{ Comorbid condition } & \multicolumn{2}{|l|}{ Patients, n (\%) } & \multirow[t]{2}{*}{ OR $(95 \% \mathrm{Cl})$} & \multirow[t]{2}{*}{$P$} \\
\hline & FM $(n=4,296)$ & No-FM $(n=583,665)$ & & \\
\hline Any musculoskeletal pain condition & $2,87 \mid(66.8)$ & $230,549(39.5)$ & $3.1(2.9,3.3)$ & $<0.0001$ \\
\hline Lupus & $93(2.2)$ & $2,752(0.5)$ & $4.7(3.8,5.8)$ & $<0.0001$ \\
\hline Diffuse diseases of connective tissue & $45(1.0)$ & $\mathrm{I}, 384(0.2)$ & $4.5(3.3,6.0)$ & $<0.0001$ \\
\hline Arthritis/other arthropathies & $\mathrm{I}, 498(34.9)$ & $103,034(17.7)$ & $2.5(2.3,2.7)$ & $<0.0001$ \\
\hline Rheumatoid arthritis & $123(2.9)$ & $5,720(1.0)$ & $3.0(2.5,3.6)$ & $<0.0001$ \\
\hline Osteoarthritis & $659(15.3)$ & $47,005(8.1)$ & $2.1(1.9,2.2)$ & $<0.0001$ \\
\hline Low-back pain & $1,390(32.4)$ & $73,543(12.6)$ & $3.3(3.1,3.5)$ & $<0.0001$ \\
\hline Back, neck pain (excluding low-back pain) & $867(20.2)$ & $38,056(6.5)$ & $3.6(3.4,3.9)$ & $<0.0001$ \\
\hline Rheumatism (excluding the back) & I,337 (31.I) & $94,477(16.2)$ & $2.3(2.2,2.5)$ & $<0.0001$ \\
\hline Other musculoskeletal pain conditions & $726(16.9)$ & $54,963(9.4)$ & $2.0(1.8,2.1)$ & $<0.000$ I \\
\hline Any neuropathic pain condition & $724(16.9)$ & $36,225(6.2)$ & $3.1(2.8,3.3)$ & $<0.0001$ \\
\hline Postherpetic neuralgia & $7(0.2)$ & $648(0.1)$ & I.5 $(0.7,3.1)$ & 0.3417 \\
\hline Carpal-tunnel syndrome & $106(2.5)$ & $6,384(I . I)$ & $2.3(1.9,2.8)$ & $<0.0001$ \\
\hline Causalgias & $72(1.7)$ & $2,802(0.5)$ & $3.5(2.8,4.5)$ & $<0.0001$ \\
\hline Neuritis radiculitis & $79(1.8)$ & $3,152(0.5)$ & $3.5(2.8,4.3)$ & $<0.0001$ \\
\hline Trigeminal neuralgia & $15(0.3)$ & $649(0.1)$ & $3.1(1.9,5.3)$ & 0.0002 \\
\hline Atypical facial pain & $7(0.2)$ & $336(0.1)$ & $2.8(1.3,6.0)$ & 0.0195 \\
\hline Phantom-limb pain & $2(<0.1)$ & $87(<0.1)$ & $3.1(0.8,12.7)$ & 0.1778 \\
\hline Autonomic neuropathies & $6(0.1)$ & $482(0.1)$ & I.7 $(0.8,3.8)$ & 0.2384 \\
\hline Mononeuritis of lower limb & $47(1.1)$ & $2,34 I(0.4)$ & $2.7(2.1,3.7)$ & $<0.0001$ \\
\hline Other polyneuropathies & $66(1.5)$ & $3,928(0.7)$ & $2.3(1.8,2.9)$ & $<0.0001$ \\
\hline $\begin{array}{l}\text { Back, neck pain with neuropathic } \\
\text { involvement (excluding low-back pain) }\end{array}$ & $462(10.8)$ & $21,082(3.6)$ & $3.2(2.9,3.5)$ & $<0.0001$ \\
\hline Any sleep disorder & $653(15.2)$ & $29,385(5.0)$ & $3.4(3.1,3.7)$ & $<0.0001$ \\
\hline Insomnia/sleep disorder, apnea & $593(13.8)$ & $26,94 I(4.6)$ & $3.3(3.0,3.6)$ & $<0.000$ I \\
\hline Restless-leg syndrome & $97(2.3)$ & $3,403(0.6)$ & $3.9(3.2,4.8)$ & $<0.0001$ \\
\hline Any mental disorder & $1,305(30.4)$ & $77,869(13.3)$ & $2.8(2.7,3.0)$ & $<0.0001$ \\
\hline Depression & $910(21.2)$ & $49,925(8.6)$ & $2.9(2.7,3.1)$ & $<0.0001$ \\
\hline Anxiety/generalized anxiety disorder & $683(15.9)$ & $39,479(6.8)$ & $2.6(2.4,2.8)$ & $<0.0001$ \\
\hline Bipolar disorder & $67(1.6)$ & $3,277(0.6)$ & $2.8(2.2,3.6)$ & $<0.0001$ \\
\hline Panic disorder & $82(1.9)$ & $3,382(0.6)$ & $3.3(2.7,4.2)$ & $<0.0001$ \\
\hline Post-traumatic stress disorder & $52(1.2)$ & $1,606(0.3)$ & $4.4(3.4,5.9)$ & $<0.0001$ \\
\hline Fatigue & $756(17.6)$ & $41,872(7.2)$ & $2.8(2.6,3.0)$ & $<0.0001$ \\
\hline Headache/migraine & $76 \mid(17.7)$ & $37,507(6.4)$ & $3.1(2.9,3.4)$ & $<0.0001$ \\
\hline Dyspareunia & $22(0.5)$ & $943(0.2)$ & $3.2(2.1,4.9)$ & $<0.0001$ \\
\hline Tinnitus & $29(0.7)$ & $3,443(0.6)$ & I.I $(0.8,1.7)$ & 0.4779 \\
\hline Chest pain & $875(20.4)$ & $57,24 I(9.8)$ & $2.4(2.2,2.5)$ & $<0.000$ I \\
\hline Temporomandibular joint disorder & $47(1.1)$ & $\mathrm{I}, 478(0.3)$ & $4.4(3.3,5.8)$ & $<0.000$ I \\
\hline Memory loss & $89(2.1)$ & $5,113(0.9)$ & $2.4(1.9,3.0)$ & $<0.0001$ \\
\hline Abnormal involuntary movements & $67(1.6)$ & $2,628(0.5)$ & $3.5(2.7,4.5)$ & $<0.0001$ \\
\hline Obesity & $466(10.8)$ & $36,553(6.3)$ & I.8 $(1.7,2.0)$ & $<0.0001$ \\
\hline Morbid obesity & $177(4.1)$ & $9,728(1.7)$ & $2.5(2.2,3.0)$ & $<0.0001$ \\
\hline Interstitial cystitis & $12(0.3)$ & $613(0.1)$ & $2.7(1.5,4.7)$ & 0.0037 \\
\hline Any gastrointestinal disorder & $1,676(39.0)$ & II4,857 (I9.7) & $2.6(2.5,2.8)$ & $<0.0001$ \\
\hline Irritable bowel syndrome & $191(4.4)$ & $7,169(1.2)$ & $3.7(3.2,4.3)$ & $<0.0001$ \\
\hline $\begin{array}{l}\text { Gastroesophageal reflux disease/gastric/ } \\
\text { duodenal/other gastrointestinal disorder }\end{array}$ & $1,626(37.8)$ & II 2,098 (19.2) & $2.6(2.4,2.7)$ & $<0.0001$ \\
\hline
\end{tabular}

Abbreviation: $\mathrm{Cl}$, confidence interval. 
diagnosis (Table 3). The presence of any musculoskeletal condition was associated with an OR of 3.1 (95\% CI 2.9, 3.3), as was the presence of any neuropathic pain condition $(95 \% \mathrm{CI}$ 2.8, 3.3). Individual comorbidities with the highest likelihood of an FM diagnosis were lupus (OR 4.7; 95\% CI 3.8, 5.8), diffuse diseases of connective tissue (OR 4.5; 95\% CI 3.3, 6.0), temporomandibular joint disorder (OR 4.4; 95\% CI 3.3, 5.8), post-traumatic stress disorder (OR 4.4; 95\% CI 3.4, 5.9), and restless-leg syndrome (OR 3.9; 95\% CI 3.2, 4.8). Among the Charlson comorbidities (Table 4), a significantly higher prevalence was observed in the FM cohort for all comorbidities except for myocardial infarction, dementia, liver disease, and hemiplegia/paraplegia; there were no malignancy-related Charlson Comorbidity Index conditions in either cohort as a result of the exclusion criterion of a cancer diagnosis. The presence of any Charlson comorbidity was associated with an almost twofold likelihood of an FM diagnosis (OR 1.9; 95\% CI 1.7, 2.0), and the Charlson comorbidity with the highest likelihood for resulting in an FM diagnosis was rheumatologic disease (OR 3.5; 95\% CI 2.8, 4.3), followed by peptic ulcer disease (OR 3.4; 95\% CI 2.8, 4.3).

\section{Health care resource utilization}

Relative to the no-FM cohort, significantly higher proportions of FM patients used each of the health care resource categories $(P<0.0001)$ except "Visits in which imaging was ordered" ( $(P=0.379)$ (Table 5$)$. The resource category with the highest likelihood of an FM diagnosis was "Visits in which diagnostic/laboratory tests were ordered," (OR 5.8; 95\% CI, 5.5, 6.3), followed by "Outpatient visits (excluding office visits)", (OR 4.8; 95\% CI 4.4, 5.1), and "Opioid prescriptions administered/ordered/written" (OR 4.6; 95\% CI 4.3, 4.9). ORs for the other resources ranged from $2.1(95 \%$ CI 2.0, 2.3) for "Hospitalizations" to 3.6 (95\% CI 3.4 3.9) for "Prescriptions administered/ordered".

Similarly, when differences between cohorts were evaluated for the number of units of resource used per patient (Table 6), the FM cohort was associated with significantly $(P<0.0001)$ greater utilization of each of the categories except for "Visits in which imaging was ordered". In both cohorts, the highest number of units used per patient was for office visits (defined as an office or clinic visit), with means of 16.8 and 11.1 office visits per patient in the FM and no-FM cohorts, respectively. The greatest difference between cohorts was for "Prescriptions administered/ordered", with 15.1 prescriptions per FM subject and 6.6 prescriptions per no-FM subject, and the greatest proportional difference was for "Opioid prescriptions administered/ordered/written", which was 3.9-fold higher per FM subject (3.5 prescriptions per subject) relative to no-FM (0.9 prescriptions per subject).

\section{Discussion}

In contrast to studies that have attempted to identify biologic factors predictive of developing FM, this study used a

Table 4 Charlson comorbidity prevalence of the evaluated cohorts during $201 \mathrm{I}$ and univariate odds ratios (ORs) for a fibromyalgia (FM) diagnosis

\begin{tabular}{|c|c|c|c|c|}
\hline \multirow[t]{2}{*}{ Comorbid condition } & \multicolumn{2}{|l|}{ Patients, n (\%) } & \multirow{2}{*}{$\begin{array}{l}\text { OR } \\
(95 \% \mathrm{CI})\end{array}$} & \multirow[t]{2}{*}{$P$} \\
\hline & $F M(n=4,296)$ & No-FM $(n=583,665)$ & & \\
\hline Any Charlson comorbidity & $\mathrm{I}, 758(40.9)$ & $158,850(27.2)$ & $1.9(1.7,2.0)$ & $<0.0001$ \\
\hline Myocardial infarction & $103(2.4)$ & $11,936(2.0)$ & $1.2(1.0,1.4)$ & 0.1133 \\
\hline Congestive heart failure & $169(3.9)$ & $17,804(3.1)$ & I.3 (I.I, I.5) & 0.0013 \\
\hline Peripheral vascular disease & $|4|(3.3)$ & $12,922(2.2)$ & $\mathrm{I} .5(\mathrm{I} .3, \mathrm{I} .8)$ & $<0.0001$ \\
\hline Cerebrovascular disease & $244(5.7)$ & $21,416(3.7)$ & $\mathrm{I} .6(\mathrm{I} .4, \mathrm{I} .8)$ & $<0.0001$ \\
\hline Dementia & $10(0.2)$ & $2,191(0.4)$ & $0.6(0.3,1.2)$ & 0.1012 \\
\hline Chronic obstructive pulmonary disease & $816(19.0)$ & $52,515(9.0)$ & $2.4(2.2,2.6)$ & $<0.0001$ \\
\hline Rheumatologic disease & $207(4.8)$ & $8,326(1.4)$ & $3.5(3.0,4.0)$ & $<0.0001$ \\
\hline Peptic ulcer disease & $80(1.9)$ & $3,195(0.5)$ & $3.4(2.8,4.3)$ & $<0.0001$ \\
\hline Mild liver disease & $28(0.7)$ & $2,849(0.5)$ & I.3 $(0.9,1.9)$ & 0.1449 \\
\hline Diabetes & $699(16.3)$ & $73,420(12.6)$ & $1.4(1.2,1.5)$ & $<0.0001$ \\
\hline Diabetes with chronic complications & $|8|(4.2)$ & $13,559(2.3)$ & $1.8(1.6,2.1)$ & $<0.0001$ \\
\hline Hemiplegia or paraplegia & $19(0.4)$ & $1,622(0.3)$ & $1.6(1.0,2.5)$ & 0.0608 \\
\hline Renal disease & $204(4.7)$ & $21,305(3.7)$ & $\mathrm{I} .3(\mathrm{I} . \mathrm{I}, \mathrm{I} .5)$ & 0.0003 \\
\hline Malignancy & $0(0.0)$ & $0(0.0)$ & - & - \\
\hline Moderate or severe liver disease & I I (0.3) & $\mathrm{I}, 232(0.2)$ & I. $2(0.7,2.2)$ & 0.5360 \\
\hline Metastatic solid tumor & $0(0.0)$ & $0(0.0)$ & - & - \\
\hline AIDS & Not reported & Not reported & - & - \\
\hline
\end{tabular}

Abbreviation: $\mathrm{Cl}$, confidence interval. 
Table 5 Frequency of health care resource use in the evaluated cohorts during $201 \mathrm{I}$

\begin{tabular}{|c|c|c|c|c|}
\hline \multirow[t]{2}{*}{ Health care resource } & \multicolumn{2}{|c|}{ Frequency, n (\%) } & \multirow{2}{*}{$\begin{array}{l}\text { OR } \\
(95 \% \mathrm{CI})\end{array}$} & \multirow[t]{2}{*}{$\mathbf{P}$} \\
\hline & $F M(n=4,296)$ & No-FM $(n=583,665)$ & & \\
\hline Emergency room visits & $\mathrm{I}, 490(34.7)$ & $105,762(18.1)$ & $2.4(2.3,2.6)$ & $<0.0001$ \\
\hline Hospitalizations & $890(20.7)$ & $63,422(10.9)$ & $2.1(2.0,2.3)$ & $<0.000$ I \\
\hline Office visits & $4,115(95.8)$ & $535,551(91.8)$ & $2.0(1.8,2.4)$ & $<0.000$ I \\
\hline Outpatient visits (excluding office visits) & $3,248(75.6)$ & $230,376(39.5)$ & $4.8(4.4,5.1)$ & $<0.000$ I \\
\hline Prescriptions administered/ordered & $2,536(59.0)$ & $165,614(28.4)$ & $3.6(3.4,3.9)$ & $<0.000$ I \\
\hline Prescriptions written & $3,802(88.5)$ & $444,400(76.1)$ & $2.4(2.2,2.6)$ & $<0.000$ I \\
\hline $\begin{array}{l}\text { Prescription pain medications administered/ordered/ } \\
\text { written* (excluding opioids) }\end{array}$ & $3,095(72.0)$ & $250,181(42.9)$ & $3.4(3.2,3.7)$ & $<0.0001$ \\
\hline Opioid prescriptions administered/ordered/written & $2,436(56.7)$ & $129,200(22.1)$ & $4.6(4.3,4.9)$ & $<0.0001$ \\
\hline Visits in which diagnostic/laboratory tests were ordered & $3,160(73.6)$ & $188,112(32.2)$ & $5.8(5.5,6.3)$ & $<0.000 \mathrm{I}$ \\
\hline Visits in which imaging was ordered & $19(0.4)$ & $2,090(0.4)$ & $1.2(0.8,1.9)$ & 0.3749 \\
\hline
\end{tabular}

Notes: *Non-opioid pain medications comprised the following classes: simple analgesics, nonsteroidal anti-inflammatory drugs, anticonvulsants, muscle relaxants, benzodiazepines, hypnotics, tricyclic antidepressants, selective serotonin reuptake inhibitors, and serotonin-norepinephrine reuptake inhibitors.

Abbreviations: $\mathrm{Cl}$, confidence interval; $\mathrm{FM}$, fibromyalgia; OR, odds ratio.

clinicoepidemiologic approach to evaluate variables associated with a clinical diagnosis based on real-world EMR data. The key findings of this study are the significant association of health care resource utilization with an FM diagnosis and the identification of the more frequently used resource categories that may potentially be useful as predictive variables. Such results are only possible with a large dataset for which there are appropriate codes available for analyses; EMR data reflect what is actually coded, providing a comprehensive selection of variables beyond demographic and clinical characteristics.

Table 6 Units per patient of health care resource use in the evaluated cohorts during $201 \mathrm{I}$

\begin{tabular}{|c|c|c|c|}
\hline \multirow[t]{2}{*}{ Health care resource } & \multicolumn{2}{|c|}{$\begin{array}{l}\text { Units per patient, } \\
\text { mean (SD) }\end{array}$} & \multirow[t]{2}{*}{$P$} \\
\hline & $\begin{array}{l}F M \\
(n=4,296)\end{array}$ & $\begin{array}{l}\text { No-FM } \\
(n=583,665)\end{array}$ & \\
\hline Emergency-room visits & $0.9(2.2)$ & $0.3(0.9)$ & $<0.0001$ \\
\hline Hospitalizations & $0.4(\mathrm{I} .0)$ & $0.2(0.6)$ & $<0.0001$ \\
\hline Office visits & $16.8(17.4)$ & II.I (I3.0) & $<0.0001$ \\
\hline $\begin{array}{l}\text { Outpatient visits } \\
\text { (excluding office visits) }\end{array}$ & $4.5(7.0)$ & $1.6(4.2)$ & $<0.0001$ \\
\hline $\begin{array}{l}\text { Prescriptions administered/ } \\
\text { ordered }\end{array}$ & I5.I (32.5) & $6.6(29.1)$ & $<0.0001$ \\
\hline Prescriptions written & $14.4(16.4)$ & $7.2(9.7)$ & $<0.0001$ \\
\hline $\begin{array}{l}\text { Prescription pain medications } \\
\text { administered/ordered/written } \\
\text { (excluding opioids) }\end{array}$ & $4.5(6.7)$ & $1.6(3.3)$ & $<0.0001$ \\
\hline $\begin{array}{l}\text { Opioid prescriptions } \\
\text { administered/ordered/written }\end{array}$ & $3.5(6.0)$ & $0.9(2.8)$ & $<0.000$ I \\
\hline $\begin{array}{l}\text { Visits in which diagnostic/ } \\
\text { laboratory tests were ordered }\end{array}$ & $3.1(4.4)$ & I.I (2.8) & $<0.0001$ \\
\hline $\begin{array}{l}\text { Visits in which imaging was } \\
\text { ordered }\end{array}$ & $0.0(0.3)$ & $0.0(0.5)$ & 0.6755 \\
\hline
\end{tabular}

Abbreviations: FM, fibromyalgia; SD, standard deviation.
The results of this study also show significant differences between the two cohorts for nearly all the demographic and clinical characteristics. Demographic differences of sex and age have previously been characterized, since the onset of FM is age-related and its prevalence is higher in females. ${ }^{4}$

While a high prevalence of comorbid conditions has also been well recognized in FM, ${ }^{10,11}$ the current analysis suggests the magnitude of these differences, both with respect to the types of comorbidities and the likelihood of their prevalence relative to no-FM subjects. With regard to the types of comorbidities, it should be noted that in addition to significant differences in comorbidities that are recognized as being associated with FM, including musculoskeletal and gastrointestinal conditions, several other comorbid conditions were observed to have a significantly higher prevalence in the FM cohort. These other comorbidities included dyspareunia, which had a more than threefold likelihood of being associated with an FM diagnosis relative to no-FM. Dyspareunia may be expected in patients with FM who may have hypersensitivity to touch, although its overall prevalence is low, possibly based on low self-report by patients and minimal questioning of sexual activity by health care providers. Other comorbidities which had a two- to fourfold increased likelihood of an FM diagnosis included chest pain, memory loss, involuntary movements, and obesity/morbid obesity. While non-cardiogenic chest pain, which may be due to pectoralis muscle spasm, can result in increased health care resource utilization, ${ }^{12}$ memory loss is a cognitive symptom that represents an important domain and is included in the 2010 ACR criteria for FM. ${ }^{4}$ Obesity, particularly morbid obesity, has been associated with decreased functional capacity in FM patients, ${ }^{13}$ and, as such, may increase the likelihood of 
diagnosis; it is not known if obesity increases the risk for FM. It should be noted that the significant differences observed between FM and no-FM for several of these comorbid conditions (eg, dyspareunia, lupus) was facilitated by both the use of EMR data and a large sample size. However, their low prevalence, even though significantly associated with FM, suggests that they are unlikely to be useful as diagnostic predictors.

A unique and important observation of this analysis is the identification of health care resource utilization categories as variables associated with an FM diagnosis. Previous studies that evaluated health care utilization in FM patients have found substantial utilization of these services. ${ }^{14,15}$ Results of the current study not only support the high utilization of health care resources in patients diagnosed with FM, but expand on the previous literature by comparing these patients with a control cohort that did not receive a diagnosis of FM. These results suggest that the statistically significant differences between these two cohorts may be of use to identify those having a higher likelihood of a future FM diagnosis. In particular, in addition to inpatient and outpatient visits and prescriptions, "Visits in which diagnostic/laboratory tests were ordered" was also significantly different between cohorts, with respect to both frequency and number of units. Indeed, "Visits in which diagnostic/laboratory tests were ordered" had the highest OR (5.8) of all the evaluated health care resource categories. This variable may be of special interest as a predictor of an FM diagnosis, since there is often a gap between FM symptom onset and diagnosis that results in high resource use in the years before diagnosis when patients may be searching for the cause of their symptoms. ${ }^{3,16,17}$ Recognition of a higher use of diagnostic/ laboratory tests may indicate those patients requiring further evaluation using a guideline-based approach for identifying the presence of FM.

\section{Strengths and limitations}

Several strengths and limitations of this study should be recognized. The use of "real-world" EMR data from multiple sites across the USA provides external validity and is an important strength of this type of analysis. Furthermore, the dataset contains data collected routinely in clinical practice, some of which are not generally available in claims databases or patient registries. The use of these data thereby enables a more comprehensive approach to identifying objective, and potentially quantifiable variables associated with an FM diagnosis that may have a direct application to clinical practice and care management. As far as we are aware, such data have not previously been used in univariate analyses to identify variables associated with FM.

On the other hand, a limitation of such database studies is that there is the potential for errors in coding or record keeping at the point of the health care provider. In this regard, as previously noted, $\geq 2$ ICD-9 codes were used to improve the accuracy of identifying the FM cohort. However, since 1.2\% of the population meeting the inclusion/exclusion criteria had a single ICD-9 code for FM, it is likely that there were patients in the no-FM cohort who were properly diagnosed with FM but were excluded from the FM cohort based on the more conservative ICD-9 inclusion criterion. Furthermore, since these databases are dependent on documentation by individual providers in the course of care delivery, clinical information such as the criteria used by each provider to diagnose FM is not routinely collected. The observational nature of the study is also a limitation, and since causality cannot be determined, all results should be considered inferential.

\section{Conclusion}

Consistent with the known epidemiology and patient-reported symptomatology of FM, demographic and clinical variables collected in clinical practice through EMR systems across the USA were identified that may be associated with an FM diagnosis. Significant differences in health care resource utilization across several resource categories were also identified, suggesting these resources are associated with an FM diagnosis. These results suggest that analysis of EMR data can help identify variables associated with FM in a real-world setting, and that further evaluation of health care resource utilization as a metric of FM diagnosis is warranted.

\section{Acknowledgments}

Editorial assistance was provided by E Jay Bienen, who was funded by Pfizer, Inc.

\section{Disclosure}

This research was sponsored by Pfizer, Inc. Elizabeth T Masters, Jack Mardekian, Birol Emir, Andrew Clair, and Max Kuhn are employees and shareholders of Pfizer, Inc., the sponsor of this study. Stuart Silverman was not financially compensated for his collaboration in this project or for the development of this paper.

\section{References}

1. Arnold LM, Clauw DJ, McCarberg BH; FibroCollaborative. Improving the recognition and diagnosis of fibromyalgia. Mayo Clin Proc. 2011; 86(5):457-464. 
2. Wolfe F, Smythe HA, Yunus MB, et al. The American College of Rheumatology 1990 Criteria for the Classification of Fibromyalgia. Report of the Multicenter Criteria Committee. Arthritis Rheum. 1990;33(2):160-172.

3. Choy E, Perrot S, Leon T, et al. A patient survey of the impact of fibromyalgia and the journey to diagnosis. BMC Health Serv Res. 2010;10:102.

4. Wolfe F, Clauw DJ, Fitzcharles MA, et al. The American College of Rheumatology preliminary diagnostic criteria for fibromyalgia and measurement of symptom severity. Arthritis Care Res (Hoboken). 2010;62(5):600-610.

5. Wolfe F, Häuser W, Hassett AL, Katz RS, Walitt BT. The development of fibromyalgia - I: examination of rates and predictors in patients with rheumatoid arthritis (RA). Pain. 2011;152(2):291-299.

6. Ablin JN, Buskila D. Predicting fibromyalgia, a narrative review: are we better than fools and children? Eur J Pain. 2014;18(8):1060-1066.

7. Häyrinen K, Saranto K, Nykänen P. Definition, structure, content, use and impacts of electronic health records: a review of the research literature. Int J Med Inform. 2008;77(5):291-304.

8. Research and analytics powered by clinical data. Boston, MA: Humedica, Inc.; 2013. Available from: http://www.humedica.com/ solutions/research/. Accessed November 3, 2014.

9. Deyo RA, Cherkin DC, Ciol MA. Adapting a clinical comorbidity index for use with ICD-9-CM administrative databases. J Clin Epidemiol. 1992;45(6):613-619.

10. White LA, Birnbaum HG, Kaltenboeck A, Tang J, Mallett D, Robinson RL. Employees with fibromyalgia: medical comorbidity, healthcare costs, and work loss. J Occup Environ Med. 2008;50(1): $13-24$
11. Lachaine J, Beauchemin C, Landry PA. Clinical and economic characteristics of patients with fibromyalgia syndrome. Clin J Pain. 2010;26(4):284-290.

12. Almansa C, Wang B, Achem SR. Noncardiac chest pain and fibromyalgia. Med Clin North Am. 2010;94(2):275-289.

13. Aparicio VA, Segura-Jiménez V, Alvarez-Gallardo IC, et al. Are there differences in quality of life, symptomatology and functional capacity among different obesity classes in women with fibromyalgia? The al-Ándalus project. Rheumatol Int. 2014;34(6):811-821.

14. Palacio A, Uribe CL, Li H, et al. Financial and clinical characteristics of fibromyalgia: a case-control comparison. Am J Manag Care. 2010; 16(5 Suppl):S118-S125.

15. Masters ET, Mardekian J, Clair A, Silverman S. Identifying predictors of a fibromyalgia diagnosis: a retrospective electronic medical record analysis [abstract]. Arthritis Rheum. 2013;65(Suppl):S52.

16. Hughes G, Martinez C, Myon E, Taieb C, Wessely S. The impact of a diagnosis of fibromyalgia on health care resource use by primary care patients in the UK. An observational study based on clinical practice. Arthritis Rheum. 2006;54(1):177-183.

17. Berger A, Sadosky A, Dukes EM, Edelsberg J, Zlateva G, Oster G. Patterns of healthcare utilization and cost in patients with newly diagnosed fibromyalgia. Am J Manag Care. 2010;16(Suppl):S126-S137.
Journal of Pain Research

\section{Publish your work in this journal}

The Journal of Pain Research is an international, peer-reviewed, open access, online journal that welcomes laboratory and clinical findings in the fields of pain research and the prevention and management of pain. Original research, reviews, symposium reports, hypothesis formation and commentaries are all considered for publication.

\section{Dovepress}

The manuscript management system is completely online and includes a very quick and fair peer-review system, which is all easy to use. Visit http://www.dovepress.com/testimonials.php to read real quotes from published authors. 